\title{
Efficient microwave-assisted synthesis of bisimides
}

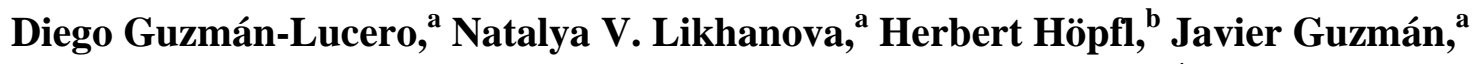 Dmitri Likhatchev, ${ }^{\mathrm{c}}$ and Rafael Martínez-Palou ${ }^{\mathrm{a},{ }^{*}}$}

\author{
${ }^{a}$ Instituto Mexicano del Petróleo. Eje Central Lázaro Cárdenas No. 152. 07730, México, \\ D.F., México \\ ${ }^{b}$ Centro de Investigaciones Químicas. Universidad Autónoma del Estado de Morelos. Av. \\ Universidad 1001. 62210, Cuernavaca, Morelos, México \\ ${ }^{c}$ Instituto de Investigaciones en Materiales. UNAM. Apdo. Postal 70-360, Coyoacán 04410, \\ México D.F., México \\ E-mail: rpalou@imp.mx
}

\begin{abstract}
A versatile, fast and efficient microwave-assisted synthetic procedure for the preparation of bisimides is described. This procedure can be applied employing either diamines (aliphatic or aromatic) or dianhydrides as reagents. The use of ionic liquids as catalysts increased the rate and yields of these reactions significantly. The products were obtained in good to excellent yields within 10 minutes of irradiation, and with high purity after a simple work-up.
\end{abstract}

Keywords: Microwave, bisimides, dianhydrides, diamines, ionic liquids

\section{Introduction}

The development of simple and versatile synthetic routes that can be applied to a wide variety of commercially available starting materials continues to be one of the most exciting topics in organic synthesis, especially when environmentally friendly methodologies are employed.

Microwave-assisted organic synthesis has been known since 1986. ${ }^{1}$ This "nonconventional" synthetic method has shown broad applications as a very efficient way to accelerate the course of many organic reactions, giving better yields and higher selectivity, lower quantities of side products and, consequently, easier work-up and purification of the products. ${ }^{2}$

Therefore, the growing interest in academic, research and industrial laboratories is not surprising and is reflected in an exponential increase in the production of scientific papers, books, ${ }^{3}$ and reviews ${ }^{4}$ related to the use of this technology.

Bisimides are heterocyclic compounds, of which some have biological activity. ${ }^{5}$ Moreover, they are synthetic precursors with application in organic synthesis, ${ }^{6}$ supramolecular chemistry, ${ }^{7}$ polymer synthesis, ${ }^{8}$ and for the development of new materials ${ }^{9}$ and molecular electronic devices. $^{10}$ 
In spite of the wide applications of bisimides, the routes for their synthesis are limited. The imidic ring formation has been carried out by condensation/dehydration reactions between anhydrides and amines or by cyclization of amic acids. ${ }^{11-13}$ Another frequently utilized method is the $N$-alkylation and $N$-arylation following the Mitsunobu or Gabriel protocols. ${ }^{14}$ However, although these procedures gave good yields for $\mathrm{N}$-arylmaleimides, the synthesis of $\mathrm{N}$ alkylmaleimides generally proceeds in low yields. In addition, these methods can not be easily generalized especially in the case of bisimides, mainly because only a few alkanols and NHbisimides are commercially available.

All routes described above have limitations: the most important are enhanced reaction times required to obtain high conversions, need of considerable volumes of toxic solvents, formation of by-products, as well as laborious work-up and purification procedures. Additionally, with certain reagents some of the above mentioned methods give only low yields, especially when these protocols are applied for bisimides instead of imides. ${ }^{11 \mathrm{a}-11 \mathrm{c}, 14 \mathrm{a}}$

Several methods for microwave-assisted imide synthesis have been published, ${ }^{15}, 16$ nevertheless, to the best of our knowledge, a general method for synthesizing bisimides with this non-conventional source of heating has not been described previously.

In this contribution, we present a fast, efficient and versatile methodology for the one-pot synthesis of bisimides, which can be applied using either dianhydrides or diamines as precursors. This process can be accelerated using ionic liquids as catalysts.

\section{Results and Discussion}

Several procedures have been reported for microwave-promoted imide syntheses, either in the presence or absence of solvent. ${ }^{15}$ The Bose methodology allows for the synthesis of an $\mathrm{N}$ alkylamide employing dimethylformamide as solvent. ${ }^{15 a, 15 b}$ Another work described the synthesis of $N$-phenylphthalimides from anhydrides and isocyanates, which are highly toxic and severe lachrymators, using dimethylacetamide as solvent. ${ }^{15 \mathrm{c}}$

The microwave-induced synthesis of imides in solvent-free conditions has been carried out by several authors. ${ }^{16}$ However, according to a reexamination of these reactions by Loupy et al., ${ }^{17}$ these methodologies can only be used in reactions between anhydrides and amines, when at least one of them is a liquid reagent, or both have a melting point very close to room temperature. Furthermore, some of these reactions require the use of expensive catalysts, ${ }^{16 \mathrm{~b},}{ }^{16 \mathrm{c}}$ and the purification of certain products must be carried out by means of chromatographic columns. ${ }^{16 a}, 16 \mathrm{c}$, $16 \mathrm{e}$.

Mallakpour and Faghihi have published several papers on microwave-assisted polycondensation reactions using bisimides functionalized with carboxyl groups and diamines or diols employing a domestic microwave. However, bisimides have been synthesized under conventional heating using acetic acid/pyridine $(3: 2)$ during $5-8$ hours of reaction time. ${ }^{8 \mathrm{~d}-8 \mathrm{k}}$ 
In most of the above-mentioned procedures, the reactions were carried out applying domestic ovens without temperature control, however, under these conditions the results are difficult to reproduce, especially when the reactions are realized with small quantities (less than 5 grams).

In our case, we initially optimized the reaction conditions to prepare $3 \mathbf{a}$ and $\mathbf{6 a}$. After some experimentation related to reagents molar ratio, solvent as well as temperature and microwave power level (data not shown), we have established a set of conditions for the synthesis of bisimides employing acetic acid as solvent. The method has been applied to the preparation of bisimides (3 and 6) derived from anhydrides (1) and diamines (2) and from dianhydrides (4) and amines (5), respectively (Scheme 1).

a)<smiles>O=C1OC(=O)c2ccccc21</smiles>

b)<smiles>O=C1OC(=O)C2CC3C(=O)OC(=O)C3CC12</smiles>

$\mathrm{R}^{2}-\mathrm{NH}_{2}$ 5

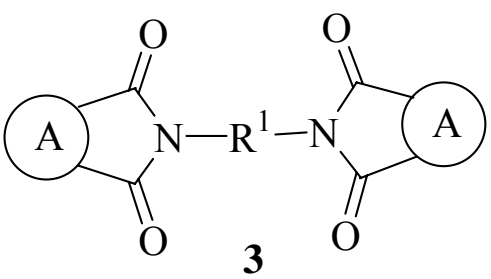

2

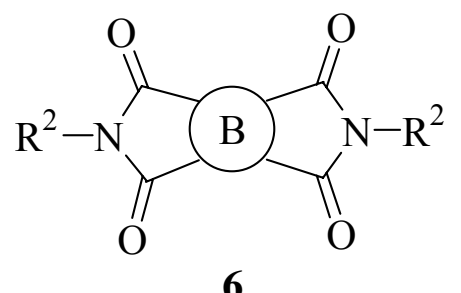

Scheme 1. Microwave-assisted synthesis of bisimides (3 and 6) from a) anhydrides (1) and diamines (2) and b) dianhydrides (4) and amines (5).

Since dianhydrides 4 are solid compounds with high melting points $\left(>200^{\circ} \mathrm{C}\right)$, such as many aromatic diamines, solvent-free procedures are not feasible for the synthesis of bisimides. ${ }^{17}$ Table 1 summarizes the compounds obtained from the reaction between anhydrides (1) and diamines (2) by irradiation at $120^{\circ} \mathrm{C}$ (power input: $300 \mathrm{~W}$ ).

The results indicate that this protocol can be used with both aliphatic (entries 1-3) and aromatic dianhydrides (entries 4-12). However, the product yields are lower in the first case, probably because of the lower thermal stability and reactivity of the succinic anhydride. For the latter, the substituent in the aromatic anhydride moiety (entries 4-12) does not have a significant influence on the reaction time. The reaction conditions are adequate for both aliphatic (entries 4 , 8,10 and 11) and aromatic diamines, but the steric hindrance of the amine substituents plays an important role for the reaction. When 2,2'-disubstituted aromatic diamines were used (entries 5 and 6), more time was required for the completion of the reaction. 
Table 1. Microwave-assisted synthesis of bisimides (3) from anhydrides (1) and diamines (2) according to Scheme 1

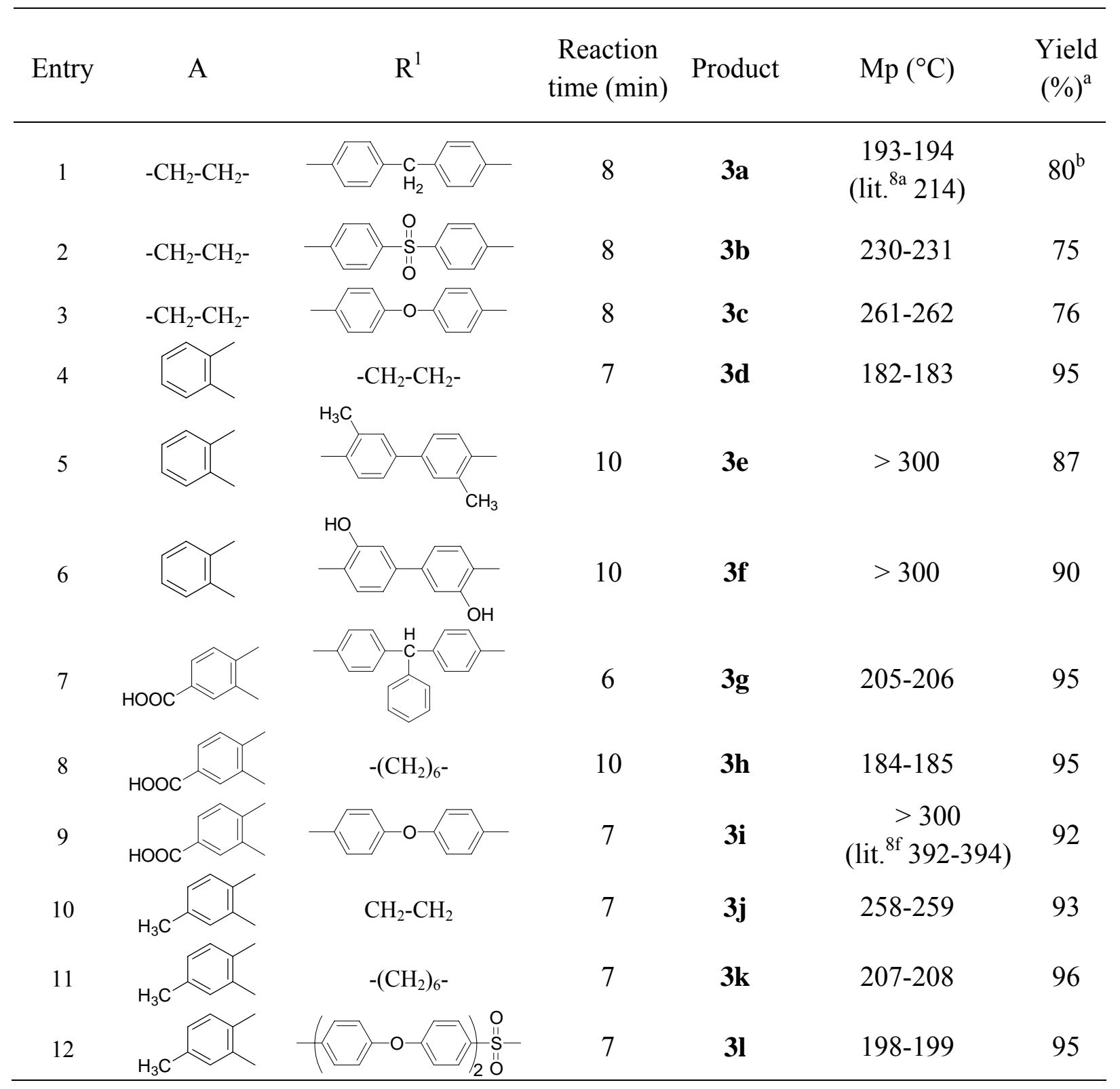

${ }^{\mathrm{a}}$ For the isolated product; ${ }^{\mathrm{b}}$ Using the same reagents, but traditional heating (oil bath at $120^{\circ} \mathrm{C}$ for 8 minutes), a mixture (40:60) of the intermediate diamide and 3a was obtained (as determined by NMR).

Table 2 shows the results of the reactions, in which dianhydrides (4) and amines (5) were employed as starting materials (irradiation at $120^{\circ} \mathrm{C}$, power input: $300 \mathrm{~W}$ ). 
Table 2. Microwave-assisted synthesis of bisimides (6) from dianhydrides (4) and amines (5) according to Scheme 1

\begin{tabular}{|c|c|c|c|c|c|c|}
\hline Run & B & $\mathrm{R}^{2}$ & $\begin{array}{l}\text { Reaction } \\
\text { time (min) }\end{array}$ & Product & $\mathrm{Mp}\left({ }^{\circ} \mathrm{C}\right)$ & $\begin{array}{l}\text { Yield } \\
(\%)^{\mathrm{a}}\end{array}$ \\
\hline 1 & & $\mathrm{CH}_{2} \mathrm{CH}_{2} \mathrm{OH}$ & 10 & $6 a$ & $\begin{array}{c}276-277 \\
\left.\text { (lit. }^{8 b} 274\right)\end{array}$ & 92 \\
\hline 2 & & & 10 & $6 b$ & $\begin{array}{c}>300 \\
\left(\text { lit. }^{11 \mathrm{~h}} 400-403\right)\end{array}$ & 93 \\
\hline 3 & & $-\mathrm{CH}_{2} \mathrm{COOH}$ & 5 & $6 c$ & $>300$ & 94 \\
\hline 4 & & & $30(4)^{b}$ & 6d & $>300$ & $37(94)^{b}$ \\
\hline 5 & & (L) $-\mathrm{CH}\left(\mathrm{CH}_{3}\right) \mathrm{COOH}$ & 5 & $6 e$ & $\begin{array}{c}261-262 \\
\left.\text { (lit. }^{8 \mathrm{e}} 262-264\right)\end{array}$ & 93 \\
\hline 6 & & & 5 & $6 f$ & $267-268$ & 97 \\
\hline 7 & & $-\mathrm{CH}_{2} \mathrm{COOH}$ & 5 & $6 g$ & $>300$ & $97^{\mathrm{c}}$ \\
\hline 8 & & $-\mathrm{CH}_{2}-\mathrm{CH}_{3}$ & 5 & $6 h$ & $161-162$ & 94 \\
\hline 9 & & & 5 & $6 \mathbf{i}$ & $\begin{array}{c}>300 \\
\left.\text { (lit. }^{8 c} 395-397\right)\end{array}$ & 97 \\
\hline 10 & & $-\left(\mathrm{CH}_{2}\right)_{17}-\mathrm{CH}_{3}$ & 7 & $6 \mathbf{j}$ & $>300$ & 96 \\
\hline 11 & & & $15(5)^{b}$ & $6 k$ & $>300$ & $78(90)^{b}$ \\
\hline 12 & & & $20(7)^{b}$ & 61 & $>300$ & $73(89)^{\mathrm{b}}$ \\
\hline
\end{tabular}

${ }^{\mathrm{a}}$ For the isolated product; ${ }^{\mathrm{b}}$ Using $10 \% \mathrm{w}$ of $[\mathrm{BMPy}] \mathrm{BF}_{4}$ as catalyst (reaction time and yield in brackets); ${ }^{\mathrm{c}}$ Using the same reagents, but traditional heating (oil bath at $120^{\circ} \mathrm{C}$ for 5 minutes), a mixture (43:57) of the intermediate diamide and $\mathbf{6} \mathbf{g}$ was obtained (as determined by NMR).

In this case, the reactions show a great versatility, since they can be performed well with a variety of amine derivatives, such as aminoalcohols (entry 1), aminophenols (entries 2 and 9), 
aminoacids (entries 3,5 and 7), including chiral example (entry 5), long alkyl chain aliphatic amines (entry 10) and anilines (entries 2, 4, 6, 9, 11 and 12). Good yields were obtained for all reactions after less than 10 minutes of irradiation time, except for $\mathbf{6 d}, \mathbf{6 k}$ and $\mathbf{6 l}$, since the nucleophilicity of the corresponding amines is diminished by the substitutent's effect on the amine group.

When aminoalcohols are used as starting materials, acetylation of the hydroxyl group can take place, if the irradiation is applied too long. For example, the reaction between pyromellitic anhydride and 2-aminoethanol (entry 1, Table 2) affords the diacetylated derivative (6aa), if the irradiation time is extended to 30 minutes. The molecular structure of this crystalline product could be determined by X-ray crystallography (see Figure 1 and Experimental Section).

With an irradiation time of 10 minutes only the 2,6-bis-(1,2-ethanodiyl)-bisimide (6a) was formed.

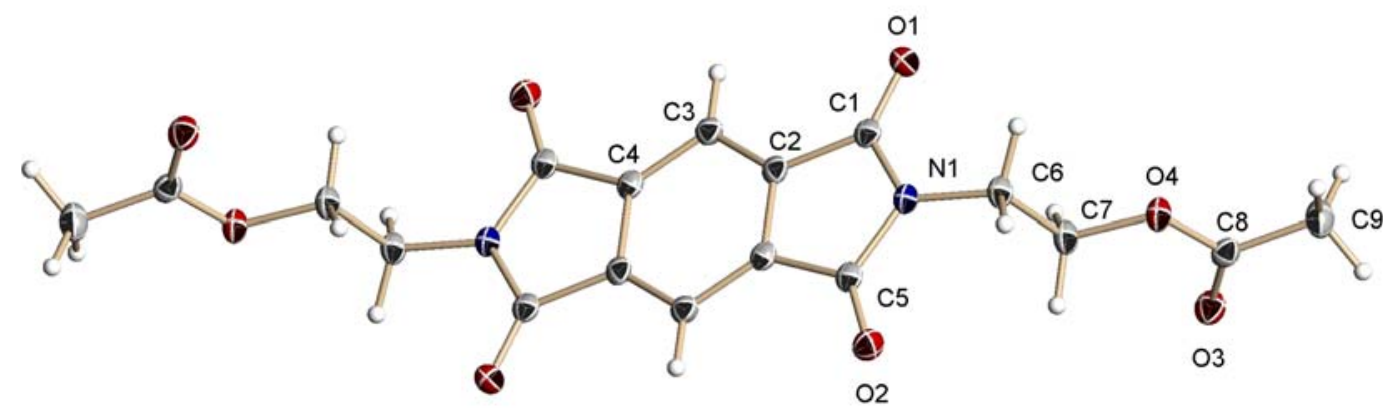

Figure 1. Perspective view of the molecular structure of compound 6aa. Thermal ellipsoids are shown at a probability level of $30 \%$.

With the objective to increase the yields and rates of the reactions that gave only moderate or low yields (entries 4, 11 and 12, Table 2), we explored the use of ionic liquids (ILs) as catalysts in these reactions.

Ionic liquids (ILs) have recently attracted much attention as solvents, cosolvents and reagents in organic synthesis, because they have negligible vapor pressures ("green solvents") and high thermal stabilities. ${ }^{18}$ Furthermore, small quantities of IL can significantly increase the rate and yields of many organic reactions. ${ }^{19}$ Due to their ionic character, ILs interact very efficiently with microwaves and their application in microwave-assisted synthesis is a new area of increasing interest. ${ }^{20,21}$

Four ILs were synthesized: 1-propyl-2,3-dimethylimidazolium iodide ([PMIM]I), 1,3dibutylimidazolium hexafluorophosphate $\left([\mathrm{DBIM}] \mathrm{PF}_{6}\right)$, 1-butylpyridinium bromide $([\mathrm{BPy}] \mathrm{Br})$, 1-butylpyridinium hexafluorophosphate $\left([\mathrm{BPy}] \mathrm{PF}_{6}\right)$, and additionally, we used 1-butyl-4methylpyridinium tetrafluoroborate ([BMPy $\left.] \mathrm{BF}_{4}\right)$ that is available from Fluka.

Figure 2 shows the kinetic effect that the above mentioned ILs have on the formation of $\mathbf{6 d}$ (entry 4, Table 2) using $10 \% \mathrm{w}$ of ILs. The reaction conversion of the crude mixture was followed by means of IR spectroscopy. 


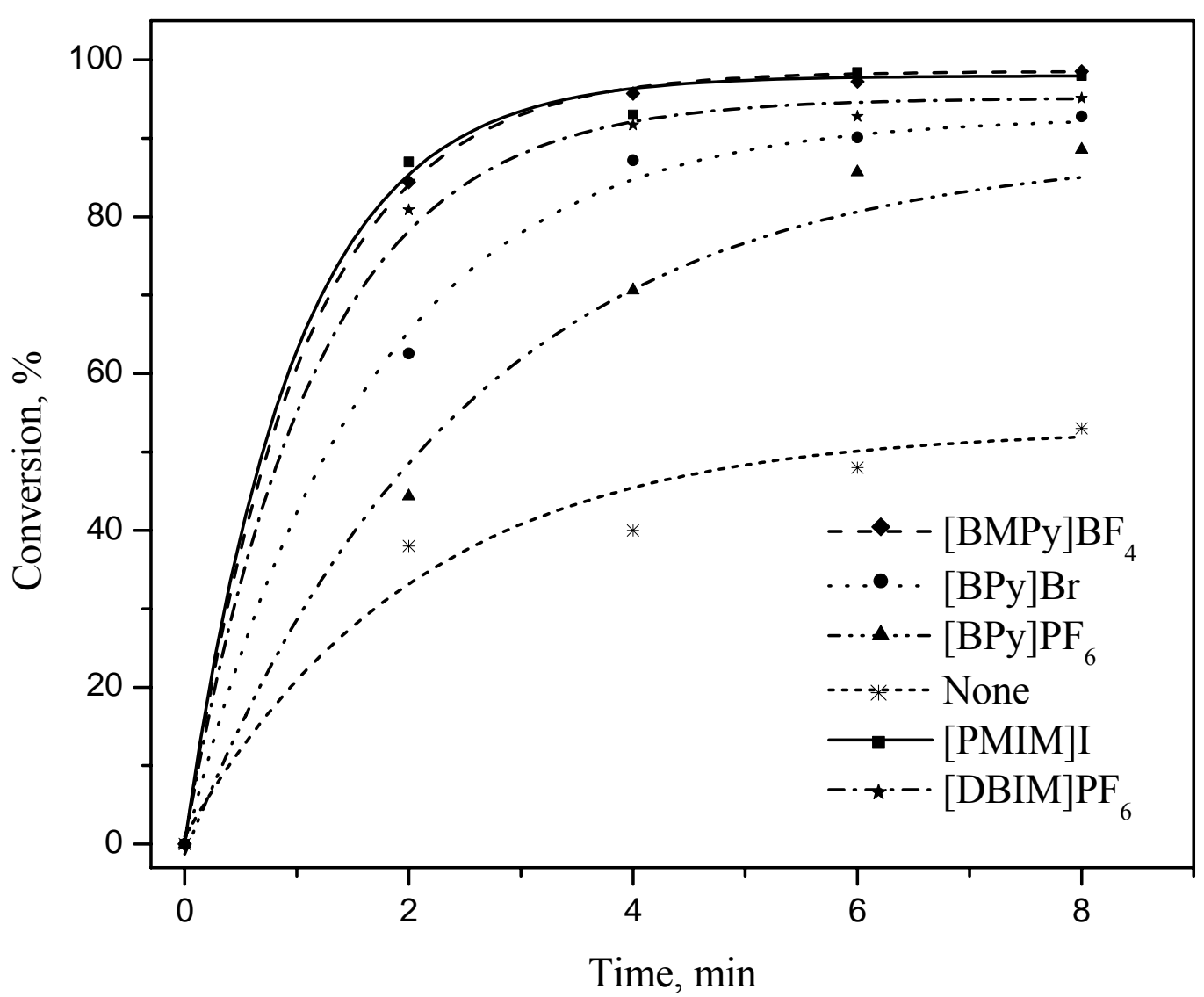

Figure 2. Kinetic effect of ionic liquids on the microwave-promoted synthesis of $\mathbf{6 d}$.

All ILs used in this study increase the reaction rate, but the best conversions and yields were obtained with [BMPy] $\mathrm{BF}_{4}$ and [PMIM]I. In order to improve also the yields of $\mathbf{6 k}$ and $\mathbf{6 l}$, the reactions were repeated in the presence of $\left[\mathrm{BMPy}_{\mathrm{BF}} \mathrm{B}_{4}\right.$ as catalyst, allowing for a significant improvement of reaction times and yields as shown in brackets in Table 2 .

The effect of the ILs on these microwave-assisted reactions is presumably twofold: first, the ILs transfer energy rapidly by ionic conduction, thus, the time required for heating the solvent is reduced (thermal effect), ${ }^{19 \mathrm{~d}}$ and, second, there is a transition state activation involving a hydrogen-bonding interaction between the imidazolium or pyridinium cation and the oxygen atom linked to the carbonyl groups of the (di)anhydrides (catalytic effect). ${ }^{21 j}$

Because the microwave-assisted reactions proceeded almost quantitatively, the work-up procedures were very simple. In most of the cases, the reaction mixture was poured in cold water and the precipitate filtered. The products were subsequently washed with water until the remaining acetic acid has been removed ( $\mathrm{pH}$ control). In those cases, in which the bisimides were partially water soluble $(\mathbf{6 c}, \mathbf{6 e}$ and $\mathbf{6 g})$, the precipitate was filtered and recrystallized from ethanol. 


\section{Experimental Section}

General Procedures. All reagents (Aldrich) were used without previous purification, except for 1-methylimidazole and pyridine, which were vacuum-distilled from $\mathrm{CaH}_{2}$ prior to use. 4,4'Diaminotriphenylmethane (2g) was synthesized by focused microwaves according to a recently described method. ${ }^{22}$

Melting points are not corrected and were measured in a Fisher Scientific apparatus equipped with a $300{ }^{\circ} \mathrm{C}$ thermometer. FT-IR spectra were registered on a Nicolet FT-IR 5DX FT spectrophotometer as $\mathrm{KBr}$ discs. Specific rotation was measured in a Perkin Elmer 241 polarimeter. ${ }^{1} \mathrm{H}$ NMR $(300 \mathrm{MHz})$ and ${ }^{13} \mathrm{C}$ NMR $(75.4 \mathrm{MHz})$ spectra were obtained with a Jeol Eclipse-300 equipment using TMS as internal standard and using the solvent specified in each case at room temperature. Mass spectra were recorded on an HP 5973 equipment with a selective mass detector. Microwave irradiations were carried out utilizing a controllable single-mode MW apparatus (MIC-I, $2450 \mathrm{MHz}$, power max. $600 \mathrm{~W}$, from SEV, Mexico). ${ }^{23}$ Open reaction tubes were used (diameter: $5 \mathrm{~cm}$, capacity $50 \mathrm{~mL}$ ), and the temperature was measured by an on-line IR detector.

General procedure for the microwave-assisted bisimide synthesis of $\mathbf{3}$ and 6 . Mixtures of anhydride 1 and diamine 2 or dianhydride $4(3.0 \mathrm{mmol})$ and amine $5(6.0 \mathrm{mmol})$, respectively, were located in pyrex reaction tubes provided with a condenser and a magnetic stirrer, overlayered with $15 \mathrm{~mL}$ of glacial acetic acid and irradiated at $120{ }^{\circ} \mathrm{C}$ (power input: $300 \mathrm{~W}$ ) for the time given in Tables 1 and 2. After cooling to room temperature the reaction mixtures were poured into $100 \mathrm{~mL}$ of cold water. The solids obtained were filtered and washed thoroughly with water to eliminate the remaining acetic acid.

A relatively large scale preparation for $3 \mathbf{k}$ and $\mathbf{6 j}$ (60 $\mathrm{mmol} \mathbf{1 k}$, and $30 \mathrm{mmol} \mathbf{2 k}$, or 30 mmol 4j and $60 \mathrm{mmol} 5 \mathbf{j}$ in $60 \mathrm{~mL}$ of solvent) in a $100 \mathrm{~mL}$ reaction tube afforded yields of $92 \%$ and $91 \%$, respectively.

In those cases, in which the products were partially water soluble, the products were precipitated at low temperature, filtered and recrystallized from ethanol.

Using ionic liquids (see Tables 2), the same procedure was employed, adding $10 \% \mathrm{~W}$ of ILs/w to the reaction mixture.

Bis-1,1'-(sulfoxydi-4,1-phenylene)-2,5-pirrolidinedione (3b). Mp 230-231 ${ }^{\circ} \mathrm{C}$ (water). ${ }^{1} \mathrm{H}$ NMR (DMSO-d 6 ) $\delta 2.52(\mathrm{~s}, 8 \mathrm{H}), 7.79(\mathrm{~d}, J=8.2 \mathrm{~Hz}, 4 \mathrm{H}), 7.86(\mathrm{~d}, J=8.2 \mathrm{~Hz}, 4 \mathrm{H}) \mathrm{ppm} .{ }^{13} \mathrm{C}$ NMR (DMSO-d $)_{6} \delta 31.7,119.5,128.9,135.6,144.1,171.5$ ppm. IR (KBr) $\tilde{v} 3355,1707,1331$, 1107, 731, $588 \mathrm{~cm}^{-1}$. MS (EI, $\left.20 \mathrm{eV}\right) \mathrm{m} / \mathrm{z}(\%) 412$ (9, $\left.\mathrm{M}^{+}\right), 330$ (78), 248 (100), 140 (31), 108 (47). Anal. calcd for $\mathrm{C}_{20} \mathrm{H}_{16} \mathrm{~N}_{2} \mathrm{O}_{6} \mathrm{~S}$ : C, 58.25; H, 3.91; N, 6.79. Found: C, 57.99; H, 3.98; N, 6.70. Bis-1,1'-(oxydi-1,4-phenylene)-2,5-pyrrolidinedione (3c). Mp 261-262 ${ }^{\circ} \mathrm{C}$ (water). ${ }^{1} \mathrm{H}$ NMR $\left(\mathrm{DMSO}_{6}\right)_{6} \delta 2.51(\mathrm{~s}, 8 \mathrm{H}), 6.93(\mathrm{~d}, J=8.2 \mathrm{~Hz}, 4 \mathrm{H}), 7.57(\mathrm{~d}, J=8.2 \mathrm{~Hz}, 4 \mathrm{H}) \mathrm{ppm} .{ }^{13} \mathrm{C}$ NMR $\left(\mathrm{DMSO}_{6} \mathrm{~d}_{6} \delta 31.0,118.6,120.5,134.8,152.3,169.8\right.$ ppm. IR (KBr) $\tilde{v} 3292,1707,1519,1188$,

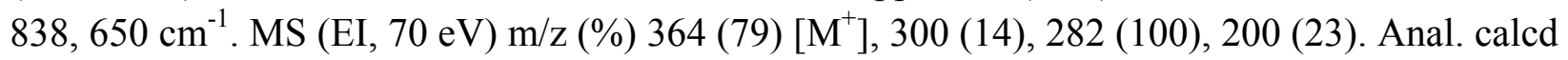
for $\mathrm{C}_{20} \mathrm{H}_{16} \mathrm{~N}_{2} \mathrm{O}_{5}$ : C, 65.93; H, 4.43; N, 7.69. Found: C, 65.99; H, 4.65; N, 7.70. 
2,2'-(1,2-Ethanediyl)bis-1H-isoindole-1,3(2H)-dione (3d). Mp 182-183 ${ }^{\circ} \mathrm{C}$ (water). ${ }^{1} \mathrm{H}$ NMR $\left(\mathrm{DMSO}_{\mathrm{d}}\right) \delta 3.85(\mathrm{~s}, 4 \mathrm{H}), 7.82(\mathrm{~m}, 8 \mathrm{H}) \mathrm{ppm} .{ }^{13} \mathrm{C}$ NMR $\left(\mathrm{DMSO}_{6}\right) \delta 36.3,39.5,123.1,131.3$, 167.8 ppm. IR (KBr) $\tilde{v}$ 3064, 2951, 1772, 1722, 1392, 1062, 881, 1062, 881, 721, $530 \mathrm{~cm}^{-1}$. MS (EI, $70 \mathrm{eV}) \mathrm{m} / \mathrm{z}(\%) 320\left(2, \mathrm{M}^{+}\right), 173$ (100), 160 (97), 77 (10). Anal. calcd for $\mathrm{C}_{18} \mathrm{H}_{12} \mathrm{~N}_{2} \mathrm{O}_{4}$ : C, 67.50; H, 3.78; N, 8.75. Found: C, 67.73; H, 3.65; N, 8.70.

2,2'-(3,3'-Dimethyl[1,1'-biphenyl]-4,4'-diyl)bis-1H-isoindole-1,3(2H)-dione (3e). Mp > 300 ${ }^{\circ} \mathrm{C}$ (water). ${ }^{1} \mathrm{H}$ NMR (DMSO-d 6$) \quad \delta 2.20(\mathrm{~s}, 6 \mathrm{H}), 7.47$ (d, $\left.J=7.4 \mathrm{~Hz}, 2 \mathrm{H}\right), 7.67$ (d, J=7.4 Hz, 2H), $7.76(\mathrm{~s}, 2 \mathrm{H}), 7.94-8.00(\mathrm{~m}, 8 \mathrm{H}) \mathrm{ppm} .{ }^{13} \mathrm{C}$ NMR (DMSO-d 6$) \delta 18.8,124.3,125.9,129.9$, 130.4, 132.4, 135.5, 137.6, 140.9, 167.7 ppm. IR (KBr) $\tilde{v} 3031,2977,2929,1782,1494,1382$, 1222, 1082, $715 \mathrm{~cm}^{-1}$. MS (EI, $\left.70 \mathrm{eV}\right) \mathrm{m} / \mathrm{z}$ (\%) 472 (100), 454 (8), 428 (9), 410 (7), 218 (9). Anal. calcd for $\mathrm{C}_{30} \mathrm{H}_{20} \mathrm{~N}_{2} \mathrm{O}_{4}$ : C, 76.26; H, 4.27; N, 5.93. Found: C, 75.94; H, 4.35; N, 5.70.

2,2'-(3,3'-Dihydroxy[1,1'-biphenyl]-4,4'-diyl)bis-1H-isoindole-1,3(2H)-dione (3f). Mp > 300 ${ }^{\circ} \mathrm{C}$ (water). ${ }^{1} \mathrm{H}$ NMR $\left(\mathrm{DMF}_{-} \mathrm{d}_{7}\right) \delta 7.24(\mathrm{dd}, J=8.0,2.0 \mathrm{~Hz}, 2 \mathrm{H}), 7.32(\mathrm{~d}, J=2.0 \mathrm{~Hz}, 2 \mathrm{H}), 7.43$ $(\mathrm{d}, J=8.0 \mathrm{~Hz}, 2 \mathrm{H}), 7.89-7.97(\mathrm{~m}, 8 \mathrm{H}), 10.3$ (brs, $2 \mathrm{H}) \mathrm{ppm} .{ }^{13} \mathrm{C}$ NMR $\left(\mathrm{DMF}-\mathrm{d}_{7}\right) \delta 115.5,118.7$, $119.8,123.9,131.6,133.1,135.2,143.2,155.5,168.0$ ppm. IR (KBr) $\tilde{v} 3042,1762,17061502$, 1384, 1111, 918, $719 \mathrm{~cm}^{-1}$. MS (EI, $\left.70 \mathrm{eV}\right) \mathrm{m} / \mathrm{z}(\%) 476\left(100, \mathrm{M}^{+}\right), 432$ (5), 488 (9). Anal. Calcd for $\mathrm{C}_{28} \mathrm{H}_{16} \mathrm{~N}_{2} \mathrm{O}_{6}$ : C, 70.59; H, 3.38; N, 5.88. Found: C, 70.92; H, 3.35; N, 5.99.

2,2'-(Methylenephenyldi[4,1-phenylene]-4,4'-diyl)bis-5-carboxy-1H-isoindole-1,3(2H)-dione (3g). Mp 205-206 ${ }^{\circ} \mathrm{C}$ (water). ${ }^{1} \mathrm{H}$ NMR (DMSO-d $) \delta 3.9$ (brs, 2H), 5.80 (s, 1H), 7.21-7.40 (m, 9H), 7.33 (d, $J=8.0 \mathrm{~Hz}, 4 \mathrm{H}), 7.43(\mathrm{~d}, J=8.0 \mathrm{~Hz}, 4 \mathrm{H}), 8.06(\mathrm{~d}, J=7.8 \mathrm{~Hz}, 2 \mathrm{H}), 8.29$ (s, 2H), $8.40(\mathrm{dd}, J=7.8,1.2 \mathrm{~Hz}, 4 \mathrm{H}) \mathrm{ppm} .{ }^{13} \mathrm{C}$ NMR $\left(\right.$ DMSO-d $\left._{6}\right) \delta 55.3,123.6,124.1,126.9,127.5$, $128.9,129.3,129.7,130.1,132.2,135.0,135.8,136.7,137.9,143.3,143.8,166.1,166.6$ ppm. IR $(\mathrm{KBr}) \tilde{v} 3173,1780,1722,1512,1377,1215,1095,729 \mathrm{~cm}^{-1}$. MS (EI, $\left.70 \mathrm{eV}\right) \mathrm{m} / \mathrm{z}(\%) 532(100$, $\mathrm{M}^{+}$- 90), 514 (9), 368 (3), 222 (5), 57 (12), 44 (56). Anal. calcd for $\mathrm{C}_{37} \mathrm{H}_{22} \mathrm{~N}_{2} \mathrm{O}_{8}$ : C, 71.38 ; $\mathrm{H}$, 3.56; N, 4.50. Found: C, 70.92; H, 3.78; N, 4.22.

2-[6-(5-Carboxy-1,3-dioxo-1,3-dihydro-2H-isoindol-2-yl)hexyl]-1,3-dioxo-5-isoindoline (3h). Mp $184-185{ }^{\circ} \mathrm{C}$ (water). $\mathrm{RMN}^{1} \mathrm{H}\left(\mathrm{DMSO}_{6}\right.$ ) $\delta 1.32$ (qi, $J=3.6 \mathrm{~Hz}, 4 \mathrm{H}$ ), 1.59 (qi, $J=6.6 \mathrm{~Hz}$, 4H), 3.5 (brs, 2H), 3.57 (t, $J=6.6 \mathrm{~Hz}, 4 \mathrm{H}), 7.95$ (d, $J=7.7 \mathrm{~Hz}, 2 \mathrm{H}), 8.19$ (s, 2H), 8.33 (dd, $J=$ 7.7, $1.1 \mathrm{~Hz}, 2 \mathrm{H}) \mathrm{ppm} .{ }^{13} \mathrm{C}$ NMR (DMSO-d 6$) \delta 25.7,27.7,37.5,122.9,123.2,132.0,134.8$, 135.1, 136.2, 165.7, 167.1 ppm. IR (KBr) v 3298, 2935, 2858, 1774, 1716, 1550, 1398, 1294,

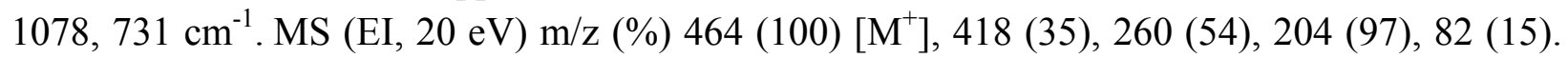
Anal. calcd for $\mathrm{C}_{24} \mathrm{H}_{20} \mathrm{~N}_{2} \mathrm{O}_{8}$ : C, 62.07; H, 4.34; N, 6.03. Found: C, 61.91; H, 4.70; N, 6.21.

2,2'-(1,2-Ethanediyl)bis-5-methyl-1H-isoindole-1,3(2H)-dione (3j). Mp 258-259 ${ }^{\circ} \mathrm{C} .{ }^{1} \mathrm{H} \mathrm{NMR}$ $\left(\mathrm{CDCl}_{3}\right) \delta 2.48(\mathrm{~s}, 6 \mathrm{H}), 3.97(\mathrm{~s}, 4 \mathrm{H}), 7.46(\mathrm{~d}, J=7.4 \mathrm{~Hz}, 2 \mathrm{H}), 7.57(\mathrm{~s}, 2 \mathrm{H}), 7.65(\mathrm{~d}, J=7.4 \mathrm{~Hz}$, 2H) ppm. ${ }^{13} \mathrm{C} \mathrm{NMR}\left(\mathrm{CDCl}_{3}\right) \delta 22.1,37.0,123.5,124.1,129.5,132.5,134.7,145.5,168.5,168.6$ ppm. IR (KBr) $\tilde{v}$ 2961, 2934, 1779, 1716, 1394, 1071, 811, $752 \mathrm{~cm}^{-1}$. MS (EI, $\left.20 \mathrm{eV}\right) \mathrm{m} / \mathrm{z}(\%)$ $348\left(6, \mathrm{M}^{+}\right), 187$ (100), 174 (97), 162 (23), 147 (17), 118 (44), 90 (13). Anal. calcd for $\mathrm{C}_{20} \mathrm{H}_{16} \mathrm{~N}_{2} \mathrm{O}_{4}$ : C, 68.96; H, 4.63; N, 8.04. Found: C, 68.81; H, 4.74; N, 8.21.

2,2'-(1,6-Hexanediyl)bis-5-methyl-1H-isoindole-1,3(2H)-dione (3k). Mp 207-208 ${ }^{\circ} \mathrm{C}$ (water). ${ }^{1} \mathrm{H}$ NMR $\left(\mathrm{CDCl}_{3}\right) \delta 1.31$ (qi, $\left.J=3.6 \mathrm{~Hz}, 4 \mathrm{H}\right), 1.60$ (qi, $\left.J=6.6 \mathrm{~Hz}, 4 \mathrm{H}\right), 2.44(\mathrm{~s}, 6 \mathrm{H}), 3.59$ (t, $J=$ 
$6.6 \mathrm{~Hz}, 4 \mathrm{H}), 7.42(\mathrm{~d}, J=7.7 \mathrm{~Hz}, 2 \mathrm{H}), 7.56(\mathrm{~s}, 2 \mathrm{H}), 7.64(\mathrm{~d}, J=7.7 \mathrm{~Hz}, 2 \mathrm{H}) \mathrm{ppm} .{ }^{13} \mathrm{C}$ NMR $\left(\mathrm{CDCl}_{3}\right) \delta 22.1,26.6,28.6,38.0,123.2,123.9,129.7,132.7,134.5,145.2,168.7,168.8$ ppm. IR $(\mathrm{KBr}) \tilde{v}$ 3046, 2922, 1776, 1716, 1512, 1377, 1087, 738, $707 \mathrm{~cm}^{-1}$. MS (EI, $\left.20 \mathrm{eV}\right) \mathrm{m} / \mathrm{z}(\%) 562$ (100) $\left[\mathrm{M}^{+}\right], 485$ (18), 460 (67), 401 (38), 326 (22), 165 (24). Anal. calcd for $\mathrm{C}_{24} \mathrm{H}_{24} \mathrm{~N}_{2} \mathrm{O}_{4}$ : C, 71.27; H, 5.98; N, 6.93. Found: C, 71.11; H, 5.76; N, 6.77.

2,2'-\{1,1'-[4,4'-(1,1'-Bisulfonylphenyl)-4,4'-diyl-dioxy]phenyl\}-bis-5-methyl-1H-isoindole-

1,3(2H)-dione (3l). Mp 198-199 ${ }^{\circ} \mathrm{C}$ (water). ${ }^{1} \mathrm{H}$ NMR $\left(\mathrm{CDCl}_{3}\right) \delta 2.56(\mathrm{~s}, 6 \mathrm{H}), 7.09-7.18(\mathrm{~m}, 8 \mathrm{H})$, $7.47(\mathrm{~d}, J=7.7 \mathrm{~Hz}, 2 \mathrm{H}), 7.59(\mathrm{~d}, J=7.7 \mathrm{~Hz}, 2 \mathrm{H}), 7.76(\mathrm{~s}, 2 \mathrm{H}), 7.84-7.93(\mathrm{~m}, 8 \mathrm{H}) \mathrm{ppm} .{ }^{13} \mathrm{C}$ NMR $\left(\mathrm{CDCl}_{3}\right) \delta 22.2,116.9,118.4,120.7,123.9,124.5,128.4,128.5,129.9,130.0,135.3$, 136.1, 146.1, 154.6, 161.6, 167.4, 167.5 ppm. IR (KBr) $\tilde{v} 3057,2921,1774,1720,1494,1371$, 1082, 825, $736 \mathrm{~cm}^{-1}$. MS (EI, $\left.20 \mathrm{eV}\right) \mathrm{m} / \mathrm{z}(\%) 446$ (5, $\mathrm{M}^{+}$- 274), 182 (55), 162 (50), 136 (100), 118 (87), 91 (63). Anal. calcd for $\mathrm{C}_{42} \mathrm{H}_{28} \mathrm{~N}_{2} \mathrm{O}_{8} \mathrm{~S}$ : C, 69.99; H, 3.92; N, 3.89. Found: C, 69.81; H, 4.10; N, 3.88.

2,6-Bis-(2-carboxymethyl)-pyrrolo[3,4-flisoindole-1,3,5,7-tetraone (6c). $\mathrm{Mp}>300{ }^{\circ} \mathrm{C}$ (ethanol). ${ }^{1} \mathrm{H}$ NMR (DMSO-d 6 ) $\delta 4.41$ (s, 4H), 8.37 (s, 2H), 13.0 (brs, 2H) ppm. ${ }^{13} \mathrm{C}$ NMR $\left(\mathrm{DMSO}_{6} \mathrm{~d}_{6} \delta 39.4,118.2,137.0,165.5,168.5\right.$ ppm. IR (KBr) $\tilde{v} 3033,1784,1718,1409,1388$, 1236, 1120, 952, 748, $621 \mathrm{~cm}^{-1}$. MS (EI, $\left.70 \mathrm{eV}\right) \mathrm{m} / \mathrm{z}(\%) 287$ (100, $\mathrm{M}^{+}$- 45), 243 (8), 158 (12), 44 (9). Anal. calcd for $\mathrm{C}_{14} \mathrm{H}_{8} \mathrm{~N}_{2} \mathrm{O}_{8}:$ C, 50.61; H, 2.43; N, 8.43. Found: C, 50.27; H, 2.34; N, 8.28. 5,5'-Carbonylbis[2-(4-nitrophenyl)]-1H-isoindole-1,3(2H)-dione (6d). $\mathrm{Mp}>300{ }^{\circ} \mathrm{C}$ (water). ${ }^{1} \mathrm{H}$ NMR (DMSO-d 6$) \delta 5.80(\mathrm{~s}, 1 \mathrm{H}), 7.20-7.40(\mathrm{~m}, 4 \mathrm{H}), 7.33(\mathrm{~d}, J=8.0 \mathrm{~Hz}, 4 \mathrm{H}), 7.43(\mathrm{~d}, J=8.0$ $\mathrm{Hz}, 4 \mathrm{H}), 8.06(\mathrm{~d}, J=8.0 \mathrm{~Hz}, 2 \mathrm{H}), 8.29(\mathrm{~s}, 2 \mathrm{H}), 8.40(\mathrm{dd}, J=8.0,3.5 \mathrm{~Hz}, 2 \mathrm{H}) \mathrm{ppm} .{ }^{13} \mathrm{C}$ NMR $\left(\right.$ DMSO-d $\left._{6}\right) \delta 55.3,123.6,124.1,126.9,127.5,128.9,129.3,129.7,130.1,132.2,135.0,135.8$, 136.7, 137.9, 143.3, 143.8, 166.1, 166.6 ppm. IR (KBr) $\tilde{v} 1784,1726,1512,1344,1211,1085$, 844, $715 \mathrm{~cm}^{-1}$. MS (EI, $\left.70 \mathrm{eV}\right) \mathrm{m} / \mathrm{z}(\%) 562\left(15, \mathrm{M}^{+}\right), 532$ (7), 442 (67), 231 (20), 117 (100), 44 (57). Anal. calcd for $\mathrm{C}_{29} \mathrm{H}_{14} \mathrm{~N}_{4} \mathrm{O}_{9}$ : C, 61.93; H, 2.51; N, 9.96. Found: C, 61.80; H, 2.50; N, 9.88. 5,5'-Carbonylbis[2-(4-methoxyphenyl)]-1H-isoindole-1,3(2H)-dione (6f). Mp 267-268 ${ }^{\circ} \mathrm{C}$ (water). ${ }^{1} \mathrm{H}$ NMR (DMSO-d 6$) \delta 3.77(\mathrm{~s}, 6 \mathrm{H}), 7.10(\mathrm{t}, J=7.4 \mathrm{~Hz}, 2 \mathrm{H}), 7.24(\mathrm{~d}, J=7.8 \mathrm{~Hz}, 2 \mathrm{H})$, $7.41(\mathrm{~d}, J=7.8 \mathrm{~Hz}, 2 \mathrm{H}), 7.51(\mathrm{t}, J=7.4 \mathrm{~Hz}, 2 \mathrm{H}), 8.18(\mathrm{~d}, J=7.7 \mathrm{~Hz}, 2 \mathrm{H}), 8.20$ (s, 2H), 8.28 (d, $J=7.7 \mathrm{~Hz}, 2 \mathrm{H}$ ) ppm. ${ }^{13} \mathrm{C}$ NMR (DMSO-d 6 ) $\delta 55.8,112.4,119.9,120.6,123.9,124.1,130.2$, $130.9,131.8,134.6,136.0,141.7,155.2,166.0,193.3$ ppm. IR (KBr) $\tilde{v} 3070,3922,2841,1782$, 1724, 1504, 1381, 1252, 1110, $725 \mathrm{~cm}^{-1}$. MS (EI, $\left.70 \mathrm{eV}\right) \mathrm{m} / \mathrm{z}(\%) 533\left(100, \mathrm{M}^{+}\right), 427$ (47), 409 (10), 103 (12). Anal. calcd for $\mathrm{C}_{31} \mathrm{H}_{20} \mathrm{~N}_{2} \mathrm{O}_{7}$ : C, 69.92; H, 3.79; N, 5.26. Found: C, 70.02; H, 3.51; N, 5.30 .

5,5'-Oxybis[2-(2-carboxymethyl)]-1H-isoindole-1,3(2H)-dione (6g). $\mathrm{Mp}>300{ }^{\circ} \mathrm{C}$ (ethanol). ${ }^{1} \mathrm{H}$ NMR (DMSO-d $\left.) \delta 4.31(\mathrm{~s}, 4 \mathrm{H}), 7.57(\mathrm{dd}, J=8.0,2.2 \mathrm{~Hz}, 2 \mathrm{H})\right), 7.63(\mathrm{~d}, J=2.2 \mathrm{~Hz}, 2 \mathrm{H})$, 7.97 (d, $J=8.0 \mathrm{~Hz}, 2 \mathrm{H}) .13 .0$ (brs, 2H) ppm. ${ }^{13} \mathrm{C}$ NMR IR (KBr) v 3464, 1772, 1708, 1392, $1274,1026,746 \mathrm{~cm}^{-1}\left(\mathrm{DMSO}_{-} \mathrm{d}_{6}\right) \delta 39.7,114.8,125.5,126.7,127.6,134.9,161.5,167.0,169.6$ ppm. MS (EI, 70 eV) m/z (\%) 424 (2, M+), 407 (13), 379 (100), 334 (35), 251 (11), 167 (18). Anal. calcd for $\mathrm{C}_{20} \mathrm{H}_{12} \mathrm{~N}_{2} \mathrm{O}_{9}$ : C, 56.61; H, 2.85; N, 6.60. Found: C, 57.00; H, 2.93; N, 6.40. 
5,5'-Oxybis(2-ethyl)-1H-isoindole-1,3(2H)-dione (6h). Mp 161-162 ${ }^{\circ} \mathrm{C}$ (water). ${ }^{1} \mathrm{H}$ NMR $\left(\mathrm{DMSO}_{\mathrm{d}}\right) \delta 1.17(\mathrm{t}, J=7.1,6 \mathrm{H}), 3.60(\mathrm{q}, J=7.1 \mathrm{~Hz}, 2 \mathrm{H}), 7.51(\mathrm{~s}, 2 \mathrm{H}), 7.53(\mathrm{~d}, J=8.8 \mathrm{~Hz}$, $2 \mathrm{H}), 7.92(\mathrm{~d}, J=8.8 \mathrm{~Hz}, 2 \mathrm{H}) \mathrm{ppm} .{ }^{13} \mathrm{C}$ NMR $\left(\mathrm{DMSO}_{-} \mathrm{d}_{6}\right) \delta 13.6,32.5,113.4,124.3,125.5$, 127.2, 134.6, 160.5, 166.7, 166.9 ppm. IR (KBr) $\tilde{v} \quad 2985,2932,1781,1705,1342,1212,1041$, $735 \mathrm{~cm}^{-1}$. MS (EI, $\left.20 \mathrm{eV}\right): \mathrm{m} / \mathrm{z}(\%)=364\left(100, \mathrm{M}^{+}\right), 349$ (93), 322 (7). Anal. calcd for $\mathrm{C}_{20} \mathrm{H}_{16} \mathrm{~N}_{2} \mathrm{O}_{5}$ : C, 65.93; H, 4.43; N, 7.69. Found: C, 65.81; H, 4.86; N, 7.42.

5,5'-Sulfoxybis(n-octadecyl)-1H-isoindole-1,3(2H)-dione (6j). Mp 121-122 ${ }^{\circ} \mathrm{C}$. ${ }^{1} \mathrm{H}$ NMR $\left(\mathrm{CDCl}_{3}\right) \delta 0.87(\mathrm{t}, J=6.6 \mathrm{~Hz}, 6 \mathrm{H}), 1.25$ (brs, 60H), 1.64 (qi, $\left.J 06.6 \mathrm{~Hz}, 4 \mathrm{H}\right), 3.69$ (t, $J=6.6 \mathrm{~Hz}$, $4 \mathrm{H}), 8.03(\mathrm{~d}, J=7.7 \mathrm{~Hz}, 2 \mathrm{H}), 8.37(\mathrm{dd}, J=7.7,1.6 \mathrm{~Hz}, 2 \mathrm{H}), 8.38(\mathrm{~s}, 2 \mathrm{H}) \mathrm{ppm} .{ }^{13} \mathrm{C}$ NMR $\left(\mathrm{CDCl}_{3}\right) \delta 14.1,22.8,26.9,28.5,29.2,29.4,29.5,29.6,29.7,32.0,38.8,122.7,124.5,133.7$, $133.8,136.5,146.1,166.2,166.4$ ppm. IR (KBr) $\tilde{v} \quad 2916,2851,1769,1705,1387,740 \mathrm{~cm}^{-1} . \mathrm{MS}$ (EI, $70 \mathrm{eV}) \mathrm{m} / \mathrm{z}(\%) 860\left(2, \mathrm{M}^{+}\right), 609$ (100), $581(25), 282$ (25). Anal. calcd for $\mathrm{C}_{52} \mathrm{H}_{80} \mathrm{~N}_{2} \mathrm{O}_{6} \mathrm{~S}: \mathrm{C}$, 72.52; H, 9.36; N, 3.25. Found: C, 72.06; H, 9.66; N, 3.07.

5,5'-Sulfoxybis(4-carboxyphenyl)-1H-isoindole-1,3(2H)-dione (6k). $\mathrm{Mp}>300{ }^{\circ} \mathrm{C}$ (water). ${ }^{1} \mathrm{H}$ NMR (DMSO-d $\left.{ }_{6}\right) \delta 7.59(\mathrm{~d}, J=8.4 \mathrm{~Hz}, 4 \mathrm{H}), 8.10(\mathrm{~d}, J=8.4 \mathrm{~Hz}, 4 \mathrm{H}), 7.59(\mathrm{~d}, J=8.4 \mathrm{~Hz}, 4 \mathrm{H})$, $8.10(\mathrm{~d}, J=8.4 \mathrm{~Hz}, 4 \mathrm{H}), 8.22(\mathrm{~d}, J=7.9 \mathrm{~Hz}, 2 \mathrm{H}), 8.64$ (d, $J=7.9 \mathrm{~Hz}, 2 \mathrm{H}), 8.69$ (s, 2H), 13.2 (brs, $2 \mathrm{H}$ ) ppm. ${ }^{13} \mathrm{C}$ NMR (DMSO-d 6 ) $\delta 123.0,125.1,127.0,130.0,130.4,133.1,134.5,135.5$, 136.2, 145.6, 166.2,165.4, 166.7 ppm. IR (KBr) $\tilde{v}$ 3094, 2825, 2677, 1778, 1728, 1585, 1365, 1151, 1060, 761, $671 \mathrm{~cm}^{-1}$. MS (EI, $\left.70 \mathrm{eV}\right) \mathrm{m} / \mathrm{z}(\%) 578$ (2, $\mathrm{M}^{+}$- 18), 477 (100), 380 (89), 379 (74), 334 (45). Anal. calcd for $\mathrm{C}_{30} \mathrm{H}_{16} \mathrm{~N}_{2} \mathrm{O}_{10} \mathrm{~S}$ : C, 60.40; H, 2.70; N, 4.70. Found: C, 59.97; H, 2.96; N, 5.00 .

5,5'-Sulfoxybis(3-carboxyphenyl)-1H-isoindole-1,3(2H)-dione (6l): $\mathrm{Mp}>300{ }^{\circ} \mathrm{C}$ (water). ${ }^{1} \mathrm{H}$ NMR (DMSO-d $)_{6} \delta$ 7.67-7.70 (m, 4H), 8.00-8.02 (m, $\left.2 \mathrm{H}\right), 8.05$ (s, 2H), 8.65 (dd, $J=7.8,1.5$ $\mathrm{Hz}, 2 \mathrm{H}), 8.70$ (s, 2H), 13.3 (brs, 2H) ppm. ${ }^{13} \mathrm{C}$ NMR (DMSO-d 6 ) $\delta 123.3,125.4,128.5,129.5$, $129.8,132.0,131.1,132.3,133.7,134.8,136.8,145.8,165.8,166.0,167.0$ ppm. IR (KBr) $\tilde{v}$ 3086, 2879, 2677, 1784, 1730, 1610, 1367, 1076, 777, $671 \mathrm{~cm}^{-1}$. MS (EI, $\left.70 \mathrm{eV}\right) \mathrm{m} / \mathrm{z}(\%) 578$ (2) $\left[\mathrm{M}^{+}-18\right], 477$ (100), 380 (75), 379 (64), 334 (20). Anal. calcd for $\mathrm{C}_{30} \mathrm{H}_{16} \mathrm{~N}_{2} \mathrm{O}_{10} \mathrm{~S}: \mathrm{C}, 60.40$; H, 2.70; N, 4.70. Found: C, 61.07; H, 2.94; N, 4.93.

The ionic liquids $[\mathrm{BPy}] \mathrm{Br},[\mathrm{BPy}] \mathrm{PF}_{6},[\mathrm{DBIM}] \mathrm{PF}_{6}$ and $[\mathrm{PMIM}] \mathrm{I}$ were synthesized as described earlier, ${ }^{24,25}$ and [BMPy] $\mathrm{PF}_{6}$ was purchased from Fluka.

\section{Crystallographic Studies}

Crystal data (for compound 6aa). $\mathrm{C}_{18} \mathrm{H}_{16} \mathrm{~N}_{2} \mathrm{O}_{8}, M=388.33$, monoclinic, $0.57 \times 0.26 \times 0.22$ $\mathrm{mm}^{3}$, colorless prismas, space group: $\mathrm{P} 2(1) / \mathrm{n}, a=8.7513(15), b=6.5717(11), c=14.961(3) \AA$, $\beta=98.596(3)^{\circ}, V=850.7(2) \AA^{3}, Z=2, \rho_{\text {calcd. }}=1.516 \mathrm{mg} / \mathrm{m}^{3}, 1585$ reflections collected $(I>2 \sigma I)$, $R_{1}=0.039, w R_{2}=0.095$.

Structure determination. X-ray diffraction data were collected on a Bruker-AXS diffractometer with a CCD area detector $\left(\lambda_{\mathrm{MoK \alpha}}=0.71073 \AA\right.$, monochromator: graphite). Frames were collected at $T=293 \mathrm{~K}$ via $\omega / \Phi$-rotation at $10 \mathrm{~s}$ per frame. ${ }^{26}$ The measured intensities were reduced to $F^{2}$ and corrected for absorption with SADABS. ${ }^{27}$ Corrections were made for Lorentz and 
polarization effects. Structure solution, refinement and data output were carried out with the SHELXTL-NT program package. ${ }^{28}$ Non hydrogen atoms were refined anisotropically. C-H hydrogen atoms were placed in geometrically calculated positions using a riding model. Figures were created with SHELXTL-NT. Crystallographic data (excluding structure factors) for the structure in this paper has been deposited at the Cambridge Crystallographic Data Center (CCDC No. 267425). Copies of the information can be obtained, free of charge, on the application to CCDC, 12 Union Road, Cambridge CB2 1EZ, UK (fax: +44-(0)1223-336033; e-mail: deposit@ccdc.cam.ac.uk or web site: http://www.ccdc.cam.ac.uk ).

\section{Acknowledgements}

We thank Q. Regina Alvarez for carrying out part of the spectroscopic analyses.

\section{References and footnotes}

1. (a) Giguere, R. J.; Bray, T. L.; Duncan, S. M.; Majetich, G. Tetrahedron Lett. 1986, 27, 4945. (b) Gedye, R.; Smith, F.; Westaway, K.; Ali, H.; Baldisera, L.; Laberge, L.; Rousell, J. Tetrahedron Lett. 1986, 27, 279.

2. Mingos, D. M. P.; Whittaker, A. G. Chemistry under Extreme on Non-Classical Conditions; van Eldrik, R.; Hubbard, C. D., Eds.; Wiley: New York, 1997, pp 479-514.

3. (a) Microwaves in Organic Synthesis; Loupy A., Ed.; Wiley-VCH: Weinheim, 2002. (b) Hayes B. L., Microwave Synthesis: Chemistry at the Speed of Light; CEM Publishing: Matthews, 2002. (c) Microwave-Assisted Organic Synthesis; Lidström P.; Tierney J. P., Eds.; Blackwell Scientific, 2005. (d) Kappe C. O.; Stadler A., Microwaves in Organic and Medicinal Chemistry; Wiley-VCH: Weinheim, 2005.

4. For recent examples, see: (a) Katritzky, A. R.; Singh, S. K. ARKIVOC 2003, (xiii), 68. (b) Nüchter, M.; Ondruschka, B.; Bonrath, W.; Gum, A. Green Chem. 2004, 6, 128. (c) Kappe, C. O. Angew. Chem. Int. Ed. 2004, 43, 6250. (d) Hayes, B. L. Aldrichimica Acta 2004, 37, 66. (e) Mavandadi, F.; Lidström, P. Curr. Topics Med. Chem. 2004, 4, 773. (e) De la Hoz, A.; Díaz-Ortiz, A.; Moreno, A. Chem. Soc. Rev. 2005, 34, 164.

5. (a) Rich, D. H.; Gesellchen, P. D.; Tong, A.; Cheung, A.; Buckner, C. K. J. Med. Chem. 1975, 18, 1004. (b) Shimazawa, R.; Miyachi, H.; Takayama, H.; Kuroda, K.; Kato, F.; Hashimoto, Y. Bio. Pharm. Bull. 1999, 22, 224.

6. (a) You, C. C.; Würthner, F. Org. Lett. 2004, 6, 2401. (b) Degenhardt, C. F.; Smith, M. D.; Shimizu, K. D. Org. Lett. 2002, 4, 723. (c) Falck-Pedersen, M. L.; Undheim, K. Tetrahedron 1996, 52, 7761. (d) Engler, T. A.; Chai, W.; Meduna, S.P. Tetrahedron Lett. 1995, 36, 2713.

7. (a) Zhang, Q.; Hamilton, D. G.; Feeder, N.; Teat S. J.; Goodman, J. M.; Sanders, J. K. New J. Chem. 1999, 23, 897. (b) Kishikawa, K.; Tsubokura, S.; Kohmoto, S.; Yamamoto, M. J. 
Org. Chem. 1999, 64, 7568. (c) Hamilton, D. G.; Sanders, J. K. M.; Davies, J. E.; Clegg, W.; Teat S. J. Chem. Comm. 1997, 9, 897. (d) Obata, Y.; Okuyama, K.; Kurihara, S.; Jinda, T. Macromolecules 1995, 28, 1547.

8. (a) Grenier-Loustalot, M. F.; Gouarderes, F.; Joubert F.; Grenier, P. Polymer 1993, 34, 3848. (b) Mary, L. J. F.; Kannan P. Eur. Polym. J. 1999, 35, 17. (c) Bruma, M.; Schulz, B.; Kopnick, T.; Robinson, J. High Perform. Polym. 2000, 12, 429. (d) Mallakpour, S.; Hajipour, A. R.; Khoee, S. J. Polym. Sci. Part A: Polym. Chem. 2000, 38, 1154. (e) Mallakpour, S.; Hajipour, A. R.; Faghihi, K. Polym. Int. 2000, 49, 1383. (f) Mallakpour, S.; Hajipour, A. R.; Faghihi, K. Eur. Polym. J. 2001, 37, 119. (g) Mallakpour, S.; Hajipour, A. R.; Habibi, S. J. Appl. Polym. Sci., 2002, 86, 2211. (h) Faghihi, K.; Hajibeygi, M. J. Appl. Polym. Sci. 2004, 92, 3447. (i) Mallakpour, S.; Rafiemanzelat, F. J. Appl. Polym. Sci. 2004, 93, 1647. (j) Faghihi, K. Macromol. Res. 2004, 12, 258. (k) Mallakpour, S.; Kowsari E. J. Appl. Polym. Sci. 2004, 91, 2992.

9. (a) Progar, D. J.; St. Clair, T. L. J. Adhesion Sci. Technol. 1991, 5, 711. (b) Würther, F.; Ahmed, S.; Thalacker, C.; Debaerdemaeker, T. Chem. Eur. J. 2002, 8, 4742. (c) Langhals, H.; Kirner, S. Eur. J. Org. Chem. 2000, 365. (d) Langhals, H.; Schonmann, G.; Feiler, L. Tetrahedron Lett. 1995, 36, 6423.

10. (a) Breeze, A. J.; Salomon, A.; Ginley, D. S.; Gregg, B. A.; Tillmann, H.; Hörhold, H.-H. Appl. Phys. Lett. 2002, 81, 3085. (b) Yakimov, A.; Forrest, S. R. Appl. Phys. Lett. 2002, 81, 3085. (c) Katz, H. E.; Bao, Z.; Gilat, S. L. Acc. Chem. Res. 2001, 34, 359. (d) Angadi, M. A.; Gosztola, D.; Wasielewski, M. R. Mater. Sci. Eng. B. 1999, 63, 191.

11. (a) Fan, L. Q.; Xu, Y. P.; Tian, H. Tetrahedron Lett. 2005, 46, 4443. (b) Chao, C.-C.; Leung, M. k.; Su, Y. O.; Chiu, K. Y.; Lin, T.-H.; Shieh, S.-J.; Lin, S. C. J. Org. Chem. 2005, 70, 4323. (c) Würthner, F.; Stepanenko, V.; Chen, Z.; Saha-Möller, C. R.; Kocher, N.; Stalke, D. J. Org. Chem. 2004, 69, 7933. (d) Wagner, T.; Davis, W. B.; Lorenz, K. B.; MichelBeyerle, M. E.; Diederichsen, U. Eur. J. Org. Chem. 2003, 3673. (e) Braish, T. F.; Fox, D. E. Synlett 1992, 12, 979. (f) Bose, A. K. Organic Synthesis 1973, 5, 973. (g) Mosher, W. A.; Chlystek, S. J. J. Heterocyclic Chem. 1972, 9, 319.

12. Reddy, P. J.; Kondo, S.; Toru, T.; Ueno, Y. J. Org. Chem. 1997, 62, 2652.

13. (a) Chang, J. H.; Park, K. M. Eur. Polym. J. 2000, 36, 2185. (b) Lewis, D. A.; Summer, J. D.; Ward, T. C.; McGrath, J. E. J. Polym. Sci. Part A: Polym. Chem. 1992, 30A, 1647.

14. (a) Hansen, J. G.; Bang, K. S.; Thorup, N.; Becher, J. Eur. J. Org. Chem. 2000, 2135. (b) Hamilton, D. G.; Lynch, D. E.; Byriel, K. A.; Kennard, C. H.; Sanders, J. K. Aust. J. Chem. 1998, 51, 441. (c) Walker, M. A. J. Org. Chem. 1995, 60, 5352. (d) Walker, M. A.; Tetrahedron Lett. 1994, 35, 665.

15. (a) Bose, A. K.; Manhas, M. S.; Ghosh, M.; Raju, V. S.; Taber, K.; Urbanczyc-Lipkowska, Z. Heterocycles 1990, 30, 741. (b) Bose, A. K.; Jayaraman, M.; Okawa, A.; Bari, S.; Robb, E. W.; Manhas, M. S. Tetrahedron Lett. 1996, 37, 6989. (c) Westaway, K. C.; Gedye, R. N. Microwave Power Electromagn. Energy 1995, 30, $219^{\circ}$ (d) Ondrus, V.; Fisera, L.; Bradac, V. ARKIVOC 2001, (v), 60. 
16. (a) Bogdal, D.; Pielichiowski, J.; Boron, A. Synlett 1996, 9, 873. (b) Chandrasekhar S.; Takhi M.; Uma G. Tetrahedron Lett. 1997, 38, 8089. (c) Chandrasekhar S.; Padmaja M. B.; Raza A. Synlett 1999, 10, 1597. (d) Seijas J. A.; Vázquez-Tato M. P.; Martínez M. M.; Nuñez-Corredoira G. J. Chem. Res. (S) 1999, 7, 420. (e) Bachín B. M.; Cuadras A. M.; Alvarez-Builla J. Synlett 2002, 2, 343. (f) Seijas J. A.; Vázquez-Tato M. P.; CarballidoReboredo R. Sixth Electronic Conference on Synthetic Organic Chemistry (ECSOC-6), 2002, E005 (www.mdpi.net).

17. Vidal, T.; Loupy, A.; Gedye, R. N. Tetrahedron 2000, 56, 5473.

18. (a) Welton, T. Chem. Rev. 1999, 99, 2071. (b) Zhao, H.; V. Malhotra, S. Aldrichimica Acta 2002, 35, 75. (c) Ionic Liquids in Synthesis, Wasserscheid P.; Keim W. Eds.; Wiley-VCH: Weinheim, 2004.

19. (a) Zhao, D.; Wu, M.; Kou, Y.; Min, E. Catalysis Today 2002, 74, 157. (b) Corma, A.; García, H. Chem. Rev. 2003, 103, 4307. (c) Olivier-Bourbigou, H.; Magna, L. J. Mol. Catal. A 2002, 182 / 183, 419. (d) Hoffmann, J.; Nüchter, M.; Ondruschka.; Wasserscheid, P. Green Chem. 2003, 5, 296.

20. (a) Leadbeater, N. E.; Torenius, H. M.; Tye, H. Comb. Chem. High Throughput Screen. 2004, 7, 511. (b) Fuentes, A.; Martínez-Palou, R.; Reyes, A.; Tamariz, J. Monatsh. Chem. 2005, 136, 177. (c) Yi, F.; Peng, Y.; Song, G. Tetrahedron Lett. 2005, 46, 3931. (d) Liao, M. C.; Duan, X. H.; Liang, Y. M. Tetrahedron Lett. 2005, 46, 3469.

21. For ILs synthesis under microwave irradiation, see: (a) Varma, R. S.; Namboodiri, V. V. Pure Appl. Chem. 2001, 73, 1309. (b) Varma, R. S.; Namboodiri, V. V. Chem. Commun. 2001, 7, 643. (c) Khadilkar, B. M.; Rebeiro, G. L. Org. Proc. Res. \& Dev. 2002, 6, 826. (d) Fraga-Dubreuil, J.; Famelart, M. H.; Bazureau, J. P. Org. Proc. Res. \& Dev. 2002, 6, 374. (e) Law, M. C.; Wong, K. Y.; Chan, T. H. Green Chem. 2002, 4, 328. (f) Deetlefs, M.; Seddon, K. R. Green Chem. 2003, 5, 181. (g) Vo-Thanh, G.; Pegot, B.; Loupy, A. Eur. J. Org. Chem. 2004, 5, 1112. (h) Kim, Y. J.; Varma, R. S. Tetrahedron Lett. 2005, 46, 1467. (i) Kim, Y. J.; Varma, R. S. J. Org. Chem. 2005, 70, 7882. (j) Kim, Y. J.; Varma, R. S. Tetrahedron Lett. 2005, 46, 7447 and ref. $20 \mathrm{~b}$.

22. Guzmán-Lucero, D.; Guzmán, J.; Likhatchev, D.; Martínez-Palou, R. Tetrahedron Lett. 2005, 46, 1119.

23. Web page: www.sevmexico.com

24. Leadbeater, N. E. ; Torenius, H. M.; Tye, H. Tetrahedron 2003, 53, 2253.

25. Vygodskii, Y. S.; Lozinskaya, E. I.; Shaplov, A. S.; Lyssenko, K. A.; Antipin, M. Y.; Urman, Y. G. Polymer 2004, 45, 5031.

26. Bruker Analytical X-ray Systems. SMART: Bruker Molecular Analysis Research Tool, Version 5.618, 2000.

27. Bruker Analytical X-ray Systems. SAINT + NT, Version 6.04, 2001.

28. Sheldrick, G. M. SHELX86, Program for Crystal Structure Solution, University of Göttingen, Göttingen (Germany), 1986; Bruker Analytical X ray Systems. SHELXTL-NT Version 6.10, 2000. 